\title{
Chronik
}

\section{Gesellschaft für empirische Philosophie}

\author{
Vortragsfolge im Wintersemester $193 \mathrm{I} / 32$
}

27. 10. 3r: Prof. Dr. Karl Korsch: Der Empirismus in der Hegelschen Philosophie.

17. Ix. 31: Prof. Dr. Walter Dubislav: Apriorismus oder Empirismus? I. 12. 31: Dr. Wilhelm Lange-Eichbaum : Das Wertproblem. 15. 12. 31: Prof. Dr. Paul Ziertmann: Empirische Pädagogik.

12. I. 32: Prof. Dr. Hans Reichenbach: Das Problem der Induktion. 19. 1. 32: Prof. Dr. Frederick Woodbridge, Columbia-Universität

Neuyork: Der Empirismus in der amerikanischen Philosophie.

26. I. 32: Prof. Dr. Ku rt Goldste in : Ober biologische Erkenntnis.

16. 2. 32: Prof. Dr. Victor Jollos: Ober den gegenwärtigen Stand des Evolutionsproblems.

1. 3. 32: Prof. Dr. Jörgen Jörgensen (Universität Kopenhagen): Probleme und Ziele der Logistik.

15. 3. 32: Priv.-Doz. Dr. A lexander Herzberg: Ist die psychoanalytische Forschungsmethode wissenschaftich brauchbar?

Weitere Vorträge werden halten: Prof. Dr. Albert Einstein, Prof. Dr. A. Dorner.

Sämtliche Vorträge fiinden an Dienstagen, $20 \mathrm{Uhr}$, im Hörsaal der zweiten Medizinischen Klinik der Charité, Berlin, Schumannstraße 2 r, statt. Mitglieder und Studenten frei. Unkostenbeitrag für Nichtmitglieder I.- RM.

$$
\text { Geschäftsstelle der Gesellschaft: }
$$

Prof. Dr. W. D u bislav, Bln.-Friedenau, Bennigsenstr. 6. H 3 Rheingau 4978.

Der Vorstand:

Prof. Dr. Friedrich Kraus. Prof. Dr. August von Parseval. Prof. Dr. Hans Reichenbach. Prof. Dr. Walter Dubislav.

Prof. Dr. Kurt Lewin. Priv.-Doz. Dr. A lexander Herzberg.

Mitgliedsbeitrag 5.- RM jährlich, bei Bezug der Zeitschrift „Erkenntnis" 20.- RM jährlich. Anmeldungen an die Geschäftsstelle.

\section{Verein Ernst Mach}

Der Verein Ernst Mach veranstaltete im Februar und März 1931 gemeinsam mit dem Wiener Volksbildungsverein die folgenden sechs Vorträge über „Probleme der Einheitswissenschaft".

I. O. Ne u rath : Einbeitswissenschaft und Empirismus der Gegenwart.

Der vom W i e n e $K$ reis vertretene Standpunkt der $E$ in he it s w is s en $s \mathrm{chaft}$ ist als Lebenserscheinung angepaßt den Bestrebungen zur Schaffung einer neuen Lebensordnung. Verwandeschaft mit dem Epikureismus im Altertum, dessen Ziel es war, die Menschen zu befreien von der Furcht vor den Göttern, vor dem Tode, vor den Schmerzen. Wissenschaft ist Werkzeug; Ziel: das Glück der Menschen. Moderne Massenbewegung fragt: wie beeinflussen soziale Umwälzungen das Glüdk? Hier wird Wissenschaft das Werkzeug der Lebensgestal- 